Integr8 currently houses data on more than 180 species of archaeans, bacteria and eukaryotes, providing information on taxonomy, genome structure and proteomics. For each species, proteins are classified according to sequence similarity, structure and function, giving the user an overview of proteome content. There is also the option of comparing this information across several species. Alternatively, if you have a particular gene or protein in mind, a simple search pulls out a wealth of information, including links to entries in gene and protein databases, structural data, key references from the published literature and a summary of expression patterns and function.

The site also provides several other useful resources. For example, the BioMart tool allows complex searches of several databases using a range of criteria, such as species name, chromosomal region, and protein structure and function. There is also access to FASTA similarity and homology searching, enabling comparisons with sequences from proteome and genome databases.

So, Integr8 truly lives up to its name, and should allow researchers from diverse fields to make the most of the increasing availability of genomic and proteomic information.

Louisa Flintoft

\title{
GENOME EVOLUTION
}

\section{Give and take}

With some parasites it's just "take... take...take" and this can include the host's genes! However, other unwelcome guests actually make generous DNA donations to their hosts.

Independent studies of Trypanosoma cruzi - the protozoan that causes Chagas disease - and the Rafflesiaceae - a family of plants that tion - show that there have been horizontal gene transfers between these parasites and their respective vertebrate and plant hosts.

Following up on the hypothesis that frequent integration of T. cruzi DNA into the host genome might underlie Chagas disease, Nadjar Nitz and colleagues extracted genomic DNA from 13 patients with Chagas disease. A probe derived from components of the T. cruzi mitochondrial DNA (kinetoplast minicircles; kDNA) was then Southern hybridized to these extracts. This hybridization showed that there were smaller fragments of kDNA than would be expected if it were present in its native form, which indicated that $\mathrm{kDNA}$ had integrated to the host genome.

Using 5'-RACE (rapid amplification of cDNA ends), the authors isoated the genomic integration sites in ech patient that they studied, identifyobserved in an experimental system?

To address this question, the authors examined rabbits that had been experimentally infected with T. cruzi for up to 3 years. These rabbits had de novo kDNA integrations, which indicated that horizontal transfer of parasite DNA to the host could be a normal part of the infection process. The rabbit and human data also indicated that $\beta$-globin loci and long interspersed nuclear elements (LINE-1) are frequent targets for $\mathrm{kDNA}$ integrations.
Interestingly, the authors went on to show that kDNA was also integrated in the genomes of the offspring of chronically infected rabbits and in chickens hatched from T. cruziinnoculated eggs. Importantly, they also found kDNA integrations in the germline of $\mathrm{F}_{2}$ chickens without persistent infections, conclusively showing vertical transfer to infectionfree progeny and so quashing any chance that their results could be artefactual.

\section{RNA WORLD}

\section{RNA stories on a mythical scale}

RNA interference (RNAi) hardly disappears off the science headlines. Most recently, the spotlight has been on Argonaute proteins - some of which are involved in the small interfering RNA (siRNA) and microRNA (miRNA) pathways. Okamura and colleagues reveal an interesting division of labour between the Argonautes in Drosophila melanogaster: Argonautel (AGO1) is required for miRNA maturation and miRNA-directed RNA cleavage, whereas Argonaute2 (AGO2) acts in the siRNA pathway.

siRNA... miRNA... the difference is more than semantic. siRNAs are the key agents for RNAi and mediate RNA destruction in a sequencespecific manner. Although miRNAs can direct RNA cleavage, they can also block translation of their targets. Both species of small RNAs carry out their functions as part of the RNA-silencing complex (RISC), a multi-protein complex that mediates RNA cleavage. Given that both types of small RNAs associate with the same RISC, do the two pathways converge at this level, or do they differ?

Prompted by previous studies in the worm, Okamura and colleagues decided to resolve the issue by looking at Ago1 and Ago2 mutants in Drosophila. They mobilized $P$-elements to delete $\mathrm{Ago} 2$ and found that it is required for RNAi in vivo and for RISC assembly; in particular, $\mathrm{AGO} 2$ is required for the unwinding of the siRNA, which is a prerequisite for siRNA-mediated cleavage. Although the RISC is associated with both siRNAs and miRNAs, Ago2 is required exclusively for the siRNA pathway.

AGO1, on the other hand, seems to be involved only in the miRNA pathway. Ago1 mutant flies die as larvae with many developmental defects, and miRNA cleavage does not occur in lysates from Ago 1 mutant embryos. In vivo analysis indicates that AGO1 directly interacts with Dicer-1, which is required for miRNA production from larger precursors, and that the role of Ago 1 is to stabilize mature miRNAs.

In mammals, siRNA and miRNA pathways seem to converge downstream of Dicer. This is not the case in other organisms, such as worms and flies. Okamura and colleagues have elegantly shown that it is Agol and 2 (as part of RISC) at 